\title{
Article \\ Case-Based Study of Metrics Derived from Instrumented Fall Risk Assessment Tests
}

\author{
Anabela Correia Martins 1,*, Joana Silva 2, António Santos ${ }^{2}$, João Madureira 2, Carlos Alcobia ${ }^{3}$, \\ Luís Ferreira ${ }^{3}$, Pedro Mendes ${ }^{3}$, Cláudia Tonelo ${ }^{3}$, Catarina Silva ${ }^{1}$, Daniela Baltazar ${ }^{1}$ and Inês \\ Sousa ${ }^{2}$ \\ 1 ESTeSC Coimbra Health School, Physiotherapy Department, Coimbra 3046-854, Portugal; \\ catarina.silva@estescoimbra.pt (C.S.); daniela.baltazar@estescoimbra.pt (D.B.) \\ 2 Fraunhofer Portugal AICOS, Porto 4200-135, Portugal; joana.silva@fraunhofer.pt (J.S.); \\ antonio.santos@fraunhofer.pt (A.S.); joao.madureira@fraunhofer.pt (J.M.); ines.sousa@fraunhofer.pt (I.S.) \\ 3 Sensing Future Technologies, Coimbra 3030-199, Portugal; carlosalcobia@sensingfuture.pt (C.A.); \\ luisferreira@sensingfuture.pt (L.F.); pedromendes@sensingfuture.pt (P.M.) \\ * Correspondence: anabelacmartins@estescoimbra.pt
}

\begin{abstract}
Purpose: National Institute for Health and Care Excellence (NICE) has recently published quality standards for assessment of fall risk and preventing further falls. According to the standards, multifactorial fall risk assessments should include: identification of falls history; analysis of gait, balance, mobility and muscle strength, among other factors. Despite being based on subjective analysis or simple timing and not being multifactorial, physiotherapists and physicians quite often use these tests as reference scales to differentiate between lower and higher risk of falling. Instrumented TUG has been recently reported to provide important additional information to the overall score. Objective: To explore a case-based approach of fall risk assessment to identify the most relevant and informative risk factors that in combination could better define a person risk profile. Materials and Methods: A multifactorial assessment of fall risk through questionnaires, standard functional tests, tests instrumented with inertial sensors, and force platforms has been studied within a group aged 55-80 years old. Different fall risk factors and fall risk assessment methods were analyzed in a case-based descriptive study. Results \& Discussion: Subjects at higher risk of falling were identified based on their detailed profiles. A set of features were obtained from the instrumented standard tests differing significantly between subjects presenting higher or lower fall risk. Therefore, instrumenting conventional tests with wearables containing inertial sensors and force platforms gives more detailed and quantitative insights. This information can be used to better define and tailor fall prevention exercises and to improve the follow-up of the evolution of the subject.
\end{abstract}

Keywords: fall risk assessment; risk of falling; force platforms; inertial sensors

\section{Introduction}

National Institute for Health and Care Excellence (NICE) has recently publised quality standards for assessment of fall risk and preventing further falls. According to the standards, multifactorial fall risk assessments should include: identification of falls history; analysis of gait, balance, mobility and muscle strength; assessement of osteoporosis risk; self-assessment of functional ability and fear of falling; vision assessment; evaluation of cognition capabilities and neurological exam; evaluation of urinary incontinence; questionnaire about home hazards; cardiovascular exam and medication revision [1-3]. Falls have an incidence of $30 \%$ of people aged over 65 years old and this number increases to $50 \%$ for those aged over 80 years old [3].

Mobility changes, namely gait and balance, impared daily activities as sit-to-stand difficulties, use of assistive device, difficulties in housing and instrumental tasks have been significantly correlated with the risk of falling [3-7]. Medical conditions as dizziness, visual acuity, rheumatic 
diseases, urinary incontinence, chronic pain, depression, polypharmacy (four or more medicines per day), and history of falls are related with increased fall risk [8].

More recently, relation between gait speed and daily activities disability has been investigated. Gait speed lower than $1.0 \mathrm{~m} / \mathrm{s}$ are associated with higher rates of hospitalization and institutionalization and the probability of benefitting from a fall prevention program is significant for persons who walk slower than $1.0 \mathrm{~m} / \mathrm{s}$ [9].

The most commonly used standard fall risk assessment tests in hospital or clinic are Timed up and Go Test (TUG) [10], Tinetti Performance Oriented Mobility Assessment (POMA) [11], Falls Risk Assessment Tool (FRAT) [12], Berg Balance Scale (BBS) [13] and FRAT-UP [14]. Despite being based on subjective analysis and not being multifactorial, physioterapists and physicians quite often use these tests as reference scales to diferentiate between groups with lower and higher risk of falling and to design appropriate preventive strategies.

More recently, approaches based on instrumented tests have been documented. Using TUG test performed with inertial wearable sensors, as reported by Xsens Technologies with seven inertial measurement units Xbus Kit, it was possible to conclude that each TUG component time can provide important additional information that otherwise cannot be obtained from the overall TUG outcome [15]. Greene et al. also reported an instrumented version of TUG with two SHIMMER kinematic sensors fixed on each shank [16]. Besides temporal parameters, they also extracted several angular velocity parameters from the gyroscope. Results suggested that this method offers an improvement over two standard falls risk assessments (TUG and BBS).

The objective of this study was to explore a case-base approach of fall risk assessment in order to identify the most relevant risk factors that in combination could better define the risk profile of a person. In addition to conventional tests and questionnaires, wearables containing inertial sensors were used to best adress mobility parameters in a group of community dwelling adults aged over 55 years.

\section{Materials and Methods}

Ten elderly volunteers (mean age $72.1 \pm 6.1$ years old) have given their informed consent and were evaluated in a physiotherapy clinic using the tests and protocols described in the following section.

This study was divided in two phases: in first day of the study (T0), the participants were assessed for their fall risk by functional tests and questionnaires. After 15 days, the participants had access to one to four sessions [17] of ICT-based fall prevention exercises during one month. Overall, the study lasted for 44 days, with a second assessment of the fall risk (T2) at the end of study by the same functional tests and questionnaires.

\subsection{Evaluation Tests and Metrics}

This section describes fall risk evaluation metrics used in this study and respective normative values.

Questionnaire on medical conditions and risk factors: this questionnaire is intended to describe personal medical history. The diseases and conditions inquired were: anxiety, depression, difficulties in sleeping, Parkinson's disease, epilepsy, rheumatic disease, chronic pain, hypertension, dizziness, urinary incontinency, previous stroke, diabetes, foot ulcers, foot pain during walking, use of assistive device, use glasses to read, use glasses for distance, hearing problems, hearing aid [18]. Questions about medications (if the person takes more than 4 medications per day) were also asked. If the person take medication for anxiety, depression or for sleeping was also registered [19].

Questionnaire on previous falls: question about past fall history (number of falls in the previous 12 months) were asked by two different interviewers [19].

Questionnaire about fear of falling: subjects were also simply asked if they had fear of falling.

Tinetti Falls Efficacy Scale (FES): is a short questionnaire presented in a smartphone that measures fear of falling during social and physical activities inside and outside home. This questionnaire was adapted to a Portuguese iconographic version and is composed by 10 questions 
rated in a four point Likert scale 1 (not concerned) to 4 (very concerned). Final score range between 0 e 100. Final scores higher than 70 are associated with higher fear of falling [20-21].

Tinetti Performance Oriented Mobility Assessment (POMA): is a task oriented test to assess gait and balance abilities, scoring each task by an ordinal scale of 0 (highest level of impairment) to 2 (independent). Final score is a sum of the result for each task of the assessment and is ranged between 0 and 28. Scores lower than 19 are associated with high fall risk and score higher than 25 are associated with low fall risk $[11,22]$.

Timed Up and Go Test (TUG) normal pace: the person is asked to start sited on a chair and when test starts, the person should stand up, walk straight for 3 meters, turn around and walk back to the chair and sit down. Final score of this test corresponds to the time needed to perform TUG test. Scores higher than 13.5 seconds were found to be associated with the occurrence of falls in a followup period of 12 months for group of 157 adults aged between 70 and 83 years old [23-24].

TUG at fast pace: same as above, but the subjects are asked to perform the test as fast as they can. Scores higher than 10 seconds were found to be associated with the occurrence of falls in a followup period of 12 months for a group of 134 adults aged between 70 and 85 years old [23, 25].

10-meters walking speed test: each individual is instructed to walk at his/her faster walking speed. It requires a $20 \mathrm{~m}$ straight path, with $5 \mathrm{~m}$ for acceleration, $10 \mathrm{~m}$ for steady-state walking, and $5 \mathrm{~m}$ for deceleration. Markers are placed at the 5 and $15 \mathrm{~m}$ positions along the path and the time to traverse is registered. Assistive devices were documented when used [26]. Whereas walking speed varies by age, gender, and anthropometrics, the range for normal walking speed is between 1.2 and $1.4 \mathrm{~m} / \mathrm{s}$. Normative values are: $0.4 \mathrm{~m} / \mathrm{s}$ indicate a probability of needing marching help at home; 0.4 to $0.8 \mathrm{~m} / \mathrm{s}$ is correlated to limited mobility; 0.8 to $1.2 \mathrm{~m} / \mathrm{s}$ subjects wander in community with some risks; $1 \mathrm{~m} / \mathrm{s}$ subjects should start a program to reduce fall risk; $>1.2 \mathrm{~m} / \mathrm{s}$ is the safe streets crossing speed [9].

Step test (ST): the ST assesses an individual's ability to place one foot onto a 7.5-cm-high step and then back down to the floor repeatedly as fast as possible for 15 seconds. The score is the number of steps completed in 15 seconds period [27]. A score of less than 10 steps in 15 seconds is associated with higher risk of falling.

Force platform exercise: postural stability was assessed by measuring the position and displacement of the centre of mass (COM) with Hercules platform, a similar system to the Wii Balance Board [28]. The subjects were instructed to stand quietly on the force platform with their arms along the body, with their feet comfortably separated [29], without shoes and eyes open for one minute. The COM position was displayed in a screen for visual feedback [30]. The objective of this test was to keep the position of the COM within an area of 5\% tolerance relatively to the initial position in the sagittal and anteroposterior directions for one minute. The percentage of the test duration is the output, as also considered in another study [28].

Questionnaire about daily physical activities: subjects were also simply asked if they practice any physical activity three or more times per week more than 30 minutes per day.

One-week step counting: each person used a smartphone with an activity monitoring algorithm and step counter enabled [31] for one week after the first session and the average number of steps per day was registered.

\subsection{Evaluations in Different Sessions}

The results of the tests TUG at normal and fast pace, POMA, 10-meters walking speed test, step test and force platform exercise were compared between T0 session and 44 days after in the T2 session.

\subsection{Instrumented TUG Test}

An instrumented version of TUG test was performed, using the 3-axial accelerometer of a smartphone placed on the pocket or fixed at the waist or at the leg and video records to collect data during the test. Instead of walking 3 meters, the test had a fixed duration of 30 seconds. Auditory cues were used to instruct the person to stand up and walk forward in the first 15 seconds and then 
turn, walk back and sit down in the last 15 seconds. For the instrumented test, a manual segmentation of the accelerometer signal was performed to obtain three segments correspondent to three phases of the TUG test: stand up, walk forward and turn around. Since it could happen that for some of the higher risk persons the given time was not enough to return to the chair, the second segment of walking and sitting down were not considered. For each segment the duration and the number of steps were also manually retrieved from the accelerometer signal and confirmed by video records. The magnitude of the accelerometer signal was computed and several statistical features were retrieved, namely, number of times the magnitude signal crosses the mean value, inter quartile range (IQR), entropy, standard deviation (Std Dev), energy, skewness and kurtosis [16, 32].

To evaluate the accelerometer-derived features that had a significant difference between the mean of higher risk and lower risk groups, a t-test for independent samples was used. Samples normality was assessed with Shapiro Wilk test. All features followed a normal distribution. Significance at a level of $95 \%$ was considered.

\section{Results}

\subsection{Dataset Description}

Descriptive statistics of the study population is provided in Table 1 . In addition to the information provided, the majority of the subjects did not use any assistive device (7 vs. 3 ) and had fear of falling (7 vs. 3), took more than 4 medicines per day (6 vs. 4), practiced less than 30 minutes of physical exercise two times per week (6 vs 4), had rheumatic problems (7 vs. 3), hypertension (7 vs. 3), foot pain while walking (9 vs. 1), used glasses (9 vs. 1). Half of population experienced dizziness and fell in the 12 months before the study. In average, during the first week, each person walked $11640 \pm 10681$ steps per day (maximum was 32421 steps and minimum was 471 steps).

In the following paragraphs a description of each person participating in the study is provided.

P1: Female, aged 80, height $1.66 \mathrm{~m}$, weight $61 \mathrm{~kg}$, BMI 22.14, takes less than 4 medicines per day, some of which were prescribed for depression. She has rheumatic diseases that cause chronic pain. Also have hypertension, diabetes and dizziness. She has visual and hearing problems. In terms of physical activity, this person is very active, reported to do physical exercises at home, as climbing stairs, walking a lot, strength exercises more than two times per week, each one more than 30 minutes.

P2: Male, aged 69, height $1.65 \mathrm{~m}$, weight $79 \mathrm{~kg}$, BMI 29.02, takes more than 4 medicines per day, some are for anxiety. He has rheumatic diseases that cause chronic pain. Also have hypertension, diabetes, urinary incontinence and dizziness. He reported to have foot pain during walking, however he is very active, attend dancing classes more than two times a week. He has hearing problems and uses glasses.

P3: Female, aged 80, height $1.50 \mathrm{~m}$, weight $70 \mathrm{~kg}$, BMI 31.11, takes more than 4 medicines per day. She has dizziness, generalized chronic pain, foot pain and unstable walking presenting the need to use a walker. She has hearing and vision problems. She reported not performing any physical activity.

P4: Female, aged 68, height $1.57 \mathrm{~m}$, weight $67 \mathrm{~kg}$, BMI 27.18, with knee prosthesis. She takes less than 4 medicines per day. She has chronic pain and foot pain, hypertension and dizziness. She uses glasses.

P5: Female, aged 65, height $1.58 \mathrm{~kg}$, weight $90 \mathrm{~kg}$, BMI 36.05, who got a total hip replacement 18 months before the study. She takes more than 4 medicines per day, some of which for depression, anxiety and for sleeping. She has rheumatism, hypertension and chronic pain and foot pain while walking, uses assistive device. In terms of physical activity, she does not perform any physical activity.

P6: Female, aged 67, height $1.59 \mathrm{~m}$, weight $82 \mathrm{~kg}$, BMI 32.44, takes more than 4 medicines per day, some for sleeping. She has rheumatism, hypertension, chronic pain and foot pain with no difficulties in standing / sitting and walking. She uses glasses. She reported that performs physical exercise more than two times per week. 
P7: Female, aged 80, height $1.57 \mathrm{~m}$, weight $58 \mathrm{~kg}$, BMI 23.53, takes more than 4 medicines per day, some are for sleeping. She has rheumatism, hypertension, dizziness, chronic pain and urinary incontinence. She has foot pain while walking, presents difficulties in walking and uses an assistive device. She also uses glasses. She does not perform any physical activity.

P8: Female, aged 65, height $1.57 \mathrm{~m}$, weight $65 \mathrm{~kg}$, BMI 26.37, takes less than 4 medicines per day, but some are for anxiety. She has chronic pain and foot pain while walking. She uses glasses. In terms of physical activity she reported that she does physical activity less than two times per week, more than 30 minutes.

P9: Female, aged 74, height 1.56 m, weight 69 kg, BMI 28.35, takes less than 4 medicines per day. She has rheumatism and chronic pain. She has vision and hearing problems. She reported that performs physical activity less than two times per week, more than 30 minutes. She is very active, despite the fact that she has Morton Neuroma (problems in the foot and chronic pain while walking).

P10: Female, aged 73, height $1.68 \mathrm{~m}$, weight $78 \mathrm{~kg}$, BMI 27.64, takes more than 4 medicines per day. She has rheumatism, diabetes, chronic pain, hypertension and urinary incontinence. She reported foot pain while walking. She uses glasses. She performs physical activity more than two times per week, each one more than 30 minutes.

Table 1: Personal information and fall risk assessment tests results for the study group in T0. Dark grey filled cells represent metric values above the thresholds indicative of fall risk, light grey filled cells represent intermediate levels of risk, for the tests in which these levels are defined.

\begin{tabular}{|c|c|c|c|c|c|c|c|c|c|c|c|}
\hline $\begin{array}{c}\text { Person } \\
\text { ID }\end{array}$ & Gender & $\begin{array}{c}\text { Age } \\
\text { (years) }\end{array}$ & $\begin{array}{c}\text { Previous } \\
\text { Falls }\end{array}$ & $\begin{array}{l}\text { Fear of } \\
\text { Falling }\end{array}$ & $\begin{array}{l}\text { FES } \\
\text { Score }\end{array}$ & POMA & $\begin{array}{c}\text { TUG } \\
\text { Normal } \\
\text { Pace (s) }\end{array}$ & $\begin{array}{l}\text { TUG } \\
\text { Fast } \\
\text { Pace } \\
\text { (s) }\end{array}$ & $\begin{array}{c}\text { Speed } \\
10-\mathrm{m} \\
\text { Test } \\
(\mathrm{m} / \mathrm{s})\end{array}$ & $\begin{array}{l}\text { Step } \\
\text { Test }\end{array}$ & $\begin{array}{c}\text { Force } \\
\text { Platform } \\
\text { (\%) }\end{array}$ \\
\hline 1 & $\mathrm{~F}$ & 80 & No & Yes & 13 & 26 & 14 & 8 & 1.5 & 18 & 58 \\
\hline 2 & M & 69 & No & Yes & 27 & 25 & 16 & 9 & 1.4 & 10 & 12 \\
\hline 3 & $\mathrm{~F}$ & 79 & Yes & Yes & 100 & 9 & 40 & 28 & 0.5 & 8 & 0 \\
\hline 4 & F & 68 & Yes & Yes & 67 & 23 & 14 & 9 & 1.2 & 11 & 12 \\
\hline 5 & $\mathrm{~F}$ & 65 & Yes & Yes & 87 & 7 & 41 & 29 & 0.5 & 9 & 10 \\
\hline 6 & $\mathrm{~F}$ & 67 & Yes & Yes & 0 & 24 & 9 & 8 & 1.4 & 12 & 25 \\
\hline 7 & $\mathrm{~F}$ & 80 & No & No & 20 & 20 & 20 & 12 & 1.1 & 7 & 10 \\
\hline 8 & $\mathrm{~F}$ & 62 & Yes & No & 23 & 25 & 9 & 6 & 1.8 & 8 & 13 \\
\hline 9 & $\mathrm{~F}$ & 74 & No & Yes & 0 & 26 & 12 & 9 & 1.3 & 12 & 30 \\
\hline 10 & $\mathrm{~F}$ & 73 & Yes & No & 20 & 24 & 11 & 8 & 1.7 & 12 & 3 \\
\hline
\end{tabular}




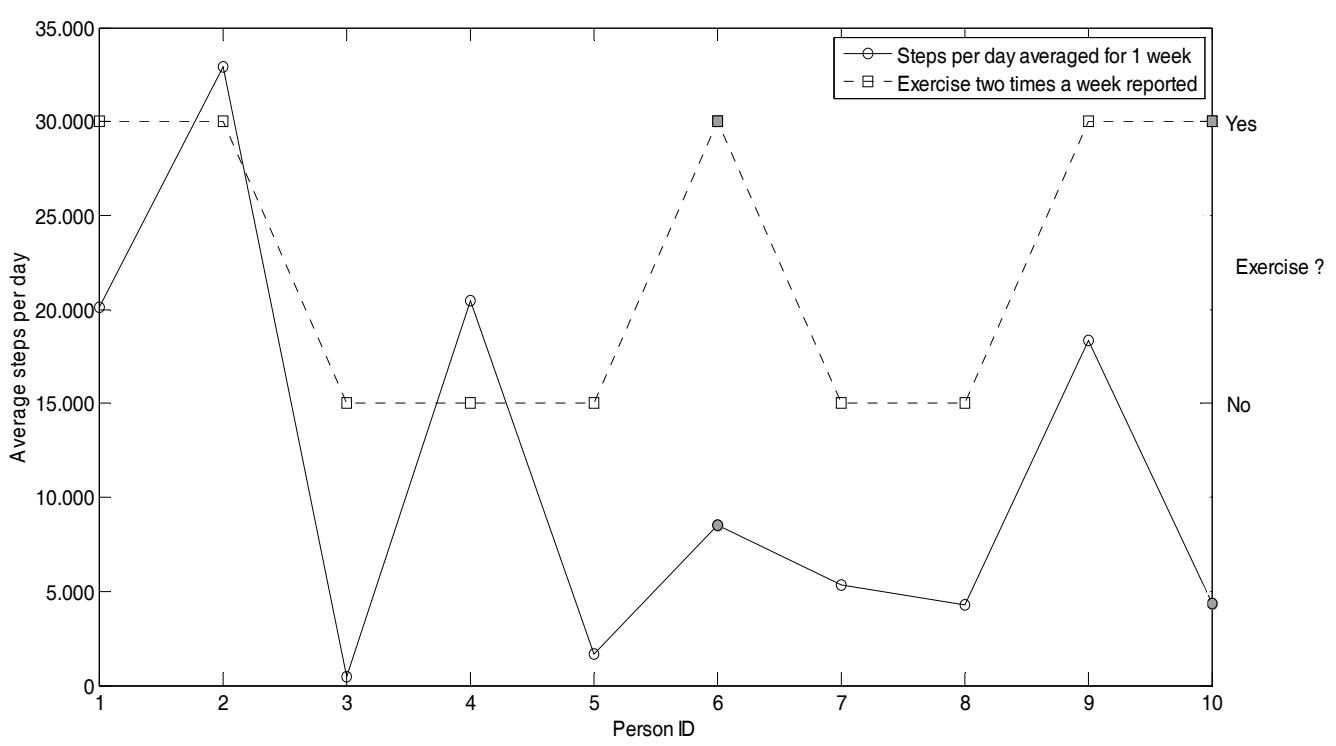

Figure 1: Comparison of steps per day averaged for one week and report of exercise at least two times a week. Inconsistences are displayed with grey filled marks.

The number of medications per day and previous falls was assessed by two different interviewers during the same session. Answers about the number of medications per day did not differ between interviewers; however, the reported falls in the previous 12 months were not the same for person ID 3, 5 and 8. FES questionnaire score and answer of the question if the person has fear of falling were not in concordance, as depicted in Table 1.

The average steps per day obtained using the smartphone step counter [31] for one week was not in concordance with answer the questionnaire about time performing physical activities per week, as shown in Figure 1.

TUG tests performed at normal and fast paces both identified the same three subjects, with times of test completion above the respective thresholds, as in higher risk of falling, in both sessions $\mathrm{T} 0$ and T2, as presented in Table 1 and Table 2. Person ID 3 and 5 have the lowest performance for the two types of TUG test, presenting scores of about 3 times the respective thresholds. Person ID 7 presents scores closer to the thresholds, which is consistent with the lower degree of impairment described in section 3.1 Dataset Description, where it can be noticed that Person ID 3 makes use of a walker to achieve mobility and Person ID 5 is recovering from a total hip arthroplasty at the time of the assessments. Three other subjects presented scores for TUG test at normal pace very close to the threshold in session 1, but in session 2 these subjects performed well below the threshold.

\subsection{Evaluations in Different Sessions}

Comparing different evaluation moments, $\mathrm{T} 0$ and $\mathrm{T} 2$, some discrepancies were noticed: TUG score between T0 and T2 decrease, as shown in Table 2. For comfortable pace TUG, an average decrease of 5.6 seconds was obtained between T0 and T2. For fast pace TUG, an average decrease of 2.4 seconds was obtained. It is worth noting that the tests results were more consistent in session 2, as in this session only the subjects identified as in higher risk of falling performed above the respective thresholds of both tests. Person ID 3 presents a higher time of completion of the TUG at fast pace than TUG at normal pace, however, given the detailed description of this case, presenting walking impairment, the difference of 3 seconds should not be valued. POMA between T0 and T2 does not differ significantly. In average, a difference of 1 point of was obtained between T0 and T2. Walking speed evaluation in 10-meters test does not differed significantly between T0 and T2. An increase of $0.1 \mathrm{~m} / \mathrm{s}$ was noticed. For the step test an average increase of 3.1 steps was obtained. An increase of 5.2 seconds was found between $\mathrm{T} 0$ and $\mathrm{T} 2$ for the force platform exercise test. 
During this trial, person ID 4, 5 and 6 were simultaneous receiving other physiotherapy modalities besides the four sessions of fall prevention program performed by the ten participants in the same clinic. However, for the high fall risk subjects, only subject ID 5, who was recovering from a hip replacement surgery, improved significantly in TUG at fast pace, step test and force platform exercise.

\subsection{Instrumented TUG Test}

According to Table 1 person ID 3, 5 and 7 were considered to be at higher risk of falling, based on the cumulative observation of TUG time higher than 13.5 seconds, POMA score lower than 25 and use of assistive device. In Table 3 and Table 4 the values of the features that best differentiate between the group of high fall risk and low fall risk for each TUG segment are presented for each subject. Among 64 features extracted from the tree segments, 14 had a p-value lower than 0.05 and are presented in Table 3 and Table 4 . For the stand segment only the time to stand and the number of times that the magnitude crosses the mean value revealed to be differentiators. For the walking segment the most relevant features were the number of steps, the standard deviation of the magnitude signal, the IQR defined as the difference between the upper and lower quartiles for an ordered set of magnitude values that measures statistical dispersion, the number of mean crossings, the signal energy defined as the sum of the squared values of magnitude over the size of the signal and the skewness and kurtosis of the signal, that are defined as the third and fourth moments of the signal. For the last segment of turn, the duration and number of steps was used and also the IQR, number of mean crossings and signal entropy.

Table 2: Personal information and fall risk assessment tests results for the study group in T2. Dark grey filled cells represent metric values above the thresholds indicative of fall risk, light grey filled cells represent intermediate levels of risk, for the tests in which these levels are defined.

\begin{tabular}{|c|c|c|c|c|c|c|c|c|c|c|c|}
\hline $\begin{array}{l}\text { Person } \\
\text { ID }\end{array}$ & Gender & $\begin{array}{c}\text { Age } \\
\text { (years) }\end{array}$ & $\begin{array}{c}\text { Previous } \\
\text { Falls }\end{array}$ & $\begin{array}{l}\text { Fear of } \\
\text { Falling }\end{array}$ & $\begin{array}{c}\text { Enrolled } \\
\text { in } \\
\text { Physio }\end{array}$ & POMA & $\begin{array}{c}\text { TUG } \\
\text { Normal } \\
\text { Pace (s) }\end{array}$ & $\begin{array}{c}\text { TUG } \\
\text { Fast } \\
\text { Pace } \\
\text { (s) }\end{array}$ & $\begin{array}{c}\text { Speed } \\
\text { 10-m } \\
\text { Test } \\
(\mathrm{m} / \mathrm{s})\end{array}$ & $\begin{array}{l}\text { Step } \\
\text { Test }\end{array}$ & $\begin{array}{c}\text { Force } \\
\text { Platform } \\
\text { (\%) }\end{array}$ \\
\hline 1 & $\mathrm{~F}$ & 80 & No & Yes & No & 27 & 10 & 8 & 1,6 & 18 & 52 \\
\hline 2 & M & 69 & No & Yes & No & 23 & 10 & 8 & 1,4 & 14 & 15 \\
\hline 3 & F & 79 & Yes & Yes & No & 7 & 21 & 24 & 0,7 & 10 & 10 \\
\hline 4 & $\mathrm{~F}$ & 68 & Yes & Yes & Yes & 23 & 10 & 9 & 1,3 & 17 & 25 \\
\hline 5 & F & 65 & Yes & Yes & Yes & 7 & 30 & 12 & 0,6 & 12 & 28 \\
\hline 6 & F & 67 & Yes & Yes & Yes & 26 & 10 & 8 & 1,4 & 11 & 26 \\
\hline 7 & $\mathrm{~F}$ & 80 & No & No & No & 20 & 14 & 11 & 1,3 & 10 & 3 \\
\hline 8 & F & 62 & Yes & No & No & 27 & 8 & 6 & 1,8 & 14 & 13 \\
\hline 9 & F & 74 & No & Yes & No & 27 & 8 & 8 & 1,4 & 19 & 23 \\
\hline 10 & F & 73 & Yes & No & No & 24 & 9 & 8 & 1,8 & 13 & 30 \\
\hline
\end{tabular}


Table 3: Accelerometer features for stand and turn segments of instrumented TUG test. Dark grey filled cells correspond to higher fall risk group.

\begin{tabular}{ccccccccc}
\hline Person Id & $\begin{array}{c}\text { Time } \\
\text { Stand }\end{array}$ & $\begin{array}{c}\text { \# Mean Cross } \\
\text { Stand }\end{array}$ & $\begin{array}{c}\text { Time } \\
\text { Turn }\end{array}$ & $\begin{array}{c}\text { \#Steps } \\
\text { Turn }\end{array}$ & $\begin{array}{c}\text { IQR } \\
\text { Turn }\end{array}$ & $\begin{array}{c}\text { \# Mean Cross } \\
\text { Turn }\end{array}$ & $\begin{array}{c}\text { Entropy } \\
\text { Turn }\end{array}$ \\
\hline $\mathbf{1}$ & 1.9 & 67 & 2.0 & 1 & 1.8 & 24 & 8.6 \\
$\mathbf{2}$ & 2.0 & 30 & 2.4 & 3 & 1.6 & 23 & 8.8 \\
$\mathbf{3}$ & 5.0 & 22 & 5.0 & 6 & 0.4 & 85 & 9.9 \\
$\mathbf{4}$ & 1.8 & 19 & 2.0 & 2 & 0.8 & 28 & 8.6 \\
$\mathbf{5}$ & 6.0 & 129 & 3.0 & 2.6 & 2 & 0.3 & 51 & 9.2 \\
$\mathbf{6}$ & 3.0 & 11 & 6.0 & 4 & 0.4 & 114 & 1.4 \\
$\mathbf{7}$ & 3.0 & 150 & 1.9 & 1 & 1.5 & 17 & 8.0 \\
$\mathbf{8}$ & 2.0 & 17 & 2.0 & 2 & 0.9 & 28 & 8.6 \\
$\mathbf{9}$ & 1.9 & 52 & 1.1 & 3 & 0.7 & 12 & 7.7 \\
\hline $\mathbf{1 0}$ & 3.9 & 0.003 & 0.002 & 0.003 & 0.008 & 0.001 & 0.003 \\
\hline p-value & 0.012 & & & & & & 29 \\
\hline
\end{tabular}

Table 4: Accelerometer features for walking segment of instrumented TUG test. Dark grey filled cells correspond to higher fall risk group.

\begin{tabular}{ccccccccc}
\hline Person Id & $\begin{array}{c}\text { \# Steps } \\
\text { Walk }\end{array}$ & Std Dev & IQR & \# Mean Cross & Energy & Skewness & \multicolumn{2}{c}{ Kurtosis } \\
& 15 & 1.9 & 2.4 & 84 & 108.2 & 0.3 & 3.3 \\
\hline $\mathbf{1}$ & 16 & 2.5 & 2.8 & 93 & 110.9 & 0.3 & 4.1 \\
\hline $\mathbf{3}$ & 12 & 1.4 & 0.6 & 141 & 102.6 & 4.2 & 29.9 \\
$\mathbf{4}$ & 14 & 2.0 & 2.2 & 73 & 106.8 & 1.3 & 4.7 \\
$\mathbf{5}$ & 8 & 1.0 & 0.5 & 152 & 100.0 & 2.6 & 17.9 \\
$\mathbf{6}$ & 17 & 2.3 & 3.0 & 74 & 106.8 & 0.8 & 3.7 \\
$\mathbf{7}$ & 12 & 1.6 & 1.0 & 122 & 104.6 & 2.5 & 14.7 \\
$\mathbf{8}$ & 20 & 2.7 & 2.3 & 112 & 115.6 & 0.9 & 5.0 \\
$\mathbf{9}$ & 18 & 2.0 & 2.3 & 114 & 105.4 & 0.8 & 4.3 \\
\hline $\mathbf{1 0}$ & 18 & 1.6 & 2.2 & 86 & 109.7 & 0.3 & 2.6 \\
\hline p-value & 0.003 & 0.013 & $2.5 \mathrm{E}-5$ & 0.003 & 0.017 & 0.0004 & 0.0003 \\
\hline
\end{tabular}

\section{Discussion \& Conclusion}

In this study we present a detailed case analysis and evaluation of several fall risk factors using different methods. The ten cases described showed different levels of physical abilities and various clinical backgrounds. Three of the subjects were identified as at higher risk of falling, since in the first evaluation (T0) their TUG at fast pace took more than 10 seconds and their TUG at normal pace more than 13.5 seconds in association with a POMA score lower than 25, gait speed in 10 meters below 1.2 meters per second, scored below 10 in the step test and below $10 \%$ in the force platform exercise and used assistive device for mobility. These three subjects were also identified as at higher fall risk based on their detailed profiles. The combination of factors increased the confidence in identifying each fall risk group.

Concerning the questionnaire answers, the results illustrate the subjectivity and difficulty in extracting reliable information when using such methods. Reporting number of previous falls is often 
used as a golden standard in fall risk assessment studies; however this question is a source of misjudgments, because it is difficult for an elderly person to remember exactly how many times he/she has fallen in a past period of time, for example, the previous year. Sometimes they tend to confuse fall with unbalance. A solution to this could be the integration of fall risk approaches with automatic fall detection solutions, such as [33] in order to have a more accurate report of previous falls. Subjects tend to report more activity than they usually do. To overcome this misevaluation, it is advisable to use a step counter for a continuous evaluation of functional capacity for at least one week [34].

Two evaluations were carried on in two different sessions with an interval of 44 days. All subjects had access to ICT-based falls prevention exercises, but the number of sessions performed was low and not sufficient to have an impact in the measured parameters. However, 3 of the subjects were enrolled in physiotherapy during the period of the study. Improvements in the metrics obtained were noticed for these 3 subjects, especially in the tests related with strength, the step test and the force platform exercise. The discrepancy of time variation between the two types of TUG test for T0 and T2 could be explained taken in account that a person has more difficulties in improve his maximum speed than his comfortable speed. Since normal speed could be improved to maximum speed in second test, but maximum speed could not be improved as much as the first one.

TUG test is very influenced by learning of test procedures and increase of confidence level, so this test is not as robust as desire for a reliable gait analysis. On other hand, POMA test revealed to be a more complete and robust approach for assessment of gait and balance. However this test is based on subjective analysis, complementary analysis could be achieved by integrating other tests such as the 10-meter walking test, step test, force platform exercises and automatic gait analysis by instrumented TUG test.

Analysis of the TUG test instrumented with inertial sensors revealed to be a useful metric to differentiate between higher and lower risk of fall among this population. As expected, persons at higher risk have mobility problems that reduce their ability to perform the TUG test on time. As so, this group takes longer to stand up and to turn. Some of the participants used an assistive device for mobility and as a consequence the time to stand also includes the time to accommodate the assistive device and start walking. During the turn, they have also to deal with the turn of the assistive device. The walking phase is also very different from a lower risk person, since higher risk person usually walk slowly and take shorter steps and normally do not raise the foot and walk alternately with an assistive device. As expected during this phase, the higher risk group takes fewer steps and has lower standard deviation of the accelerometer magnitude.

Regarding the force platform exercise, the results were slightly improved in T2 for 6 subjects. The subjects presenting scores associated with lower risk of falling consistently in the various tests, scored over $10 \%$ (maintaining the sagittal and anteroposterior position for at least 10 seconds) in this exercise. However, there were some low percentage values probably due to the test performance which may be difficult for some individuals to maintain the initial position within a $5 \%$ tolerance. It must be noted that this test was not performed as a standard balance test, instead it was used for assessment of the standing posture.

By collecting accelerometry and force platform metrics during standard fall risk evaluation tests it is possible to obtain more detailed and quantitative information. This information could be used to better define and tailor fall prevention exercises in order to revert or control some fall risk factors that could be preventable.

In this case-based study, the group was homogeneous. All the individuals have a serious number of risk factors for falling, like 4 or more medicines per day, foot pain, chronic pain, vision and hearing problems, rheumatism, dizziness and age. This means that all the individuals are at high risk of falling.

In future research, it's important to study other individuals with different risk profiles, in order to address all kinds of fall risk, from the lowest to highest. This will allow us to create individual and tailored intervention plans according to these profiles. 
In conclusion, the fall risk assessment methods should evaluate multiple fall risk factors and be as detailed and objective as possible, avoiding to rely on personal reports. Additional tests for assessment fall risk with metrics extracted from inertial sensors and force platforms could be an effective strategy to obtain more insights about mobility condition in a quantitative way. In the future, fall risk assessment in combination with fall detection and fall prevention should be encouraged on a daily analysis and ICT may play a crucial role in delivering the appropriate solutions.

Acknowledgments: Financial support from PT2020 number 003464, FallSensing: Technological solution for fall risk screening and falls prevention, co-funded by European Union (EU) by the European Structural Investment Funds (ESIF) under of the COMPETE 2020 (Operational Program for Competitiveness and Internationalization).

Author Contributions: ACM, JS, IS: Study concept and design, analysis and interpretation of the data, drafting of manuscript, critical revision. JS, AS, JM, CS, DB, CT, CA, LF and PM: collecting part of the experimental data, analysis and interpretation of the data, writing of manuscript. All the authors have read and approved the final manuscript.

Conflicts of Interest: The authors declare no conflict of interest.

\section{Abbreviations}

The following abbreviations are used in this manuscript:

NICE: National Institute for Health and Care Excellence

TUG: Timed up and Go Test

POMA: Performance Oriented Mobility Assessment

FRAT: Falls Risk Assessment Tool

BBS: Berg Balance Scale

ICT: Information and Communication Technology

FES: Falls Efficacy Scale

ST: Step test

COM: Centre of mass

IQR: Inter quartile range

Std Dev: Standard deviation

BMI: Body Mass Index

\section{References}

1. National Institute for Health and Care Excellence (NICE). 2015. Falls in older people - Quality standard. 2015. Available online: nice.org.uk/guidance/qs86 (accessed on the 11 February 2016).

2. Salarian, A.; Horak, F.B.; Zampieri, C.; CarlsonKuhta, P.; Nutt, J.G. \& Aminian, K. iTUG, a Sensitive and Reliable Measure of Mobility. IEEE Trans Neural Syst Rehabil Eng 2010 Jun, 18 (3), 303-310, DOI:10.1109/TNSRE.2010.2047606.

3. NICE guidelines. Falls in older people: assessing risk and prevention. 2013. Available online: https://www.nice.org.uk/guidance/cg161/chapter/recommendations (accessed on 20 October 2015).

4. Moreland, J.; Richardson, J.; Chan, D.H.; O'Neill, J.; Bellissimo, A.; Grum, R.M. \& Shanks, L. Evidence-Based Guidelines for the Secondary Prevention of Falls in Older Adults. Gerontology 2003, 49, 93-116, DOI:10.1159/000067948.

5. Moreland, J.D.; Richardson, J.A.; Goldsmith, C.H. \& Clase, C.M. Muscle Weakness and Falls in Older Adults: A Systematic Review and Meta-Analysis: muscle weakness and falls in older adults. J Am Geriatr Soc 2004, 52, 1121-1129, DOI:10.1111/j.1532-5415.2004.52310.x.

6. Muir, S.W.; Berg, K.; Chesworth, B.; Klar, N. \& Speechley, M. Application of a fall screening algorithm stratified fall risk but missed preventive opportunities in community-dwelling older adults: a prospective study. J Geriatr Phys Ther 2010, 33, 165-172, DOI:10.1097/JPT.0b013e3181ff23cc. 
7. Oliver, D.; Daly, F.; Martin, F.C. \& McMurdo, M.E.T. Risk factors and risk assessment tools for falls in hospital in-patients: a systematic review. Age Ageing 2004, 33, 122-130, DOI:10.1093/ageing/afh017.

8. Gillespie, L.D.; Robertson, M.C.; Gillespie, W.J.; Sherrington, C.; Gates, S.; Clemson, L.M. \& Lamb, S.E. Interventions for preventing falls in older people living in the community. Cochrane Database Syst Rev, 2012, 9, CD007146, DOI:10.1002/14651858.CD007146.pub3.

9. Fritz, S. \& Lusardi, M. White Paper: “Walking Speed: the Sixth Vital Sign”. J Geriatr Phys Ther 2009, 32 (2), 46-49. Available online: http://tinyurl.com/hao4ton (accessed on the 20 January 2016).

10. Sprint, G.; Cook, D. J. \& Weeks, D. L. Toward Automating Clinical Assessments: A Survey of the Timed Up and Go. Rev Biomed Eng, 2015, 8, 64-77, DOI:10.1109/RBME.2015.2390646.

11. Faber, M.J.; Bosscher, R.J. \& van Wieringen, P.C. Clinimetric Properties of the Performance-Oriented Mobility Assessment. Phys Ther 2006, 86, 944-954, DOI:10.1109/RBME.2015.2390646 6.

12. Nandy, S.; Parsons, S.; Cryer, C.; Underwood, M.; Rashbrook, E.; Carter, Y.; Eldridge, S.; Close, J.; Skelton, D.; Taylor, S. \& Feder, G. Development and preliminary examination of the predictive validity of the Falls Risk Assessment Tool (FRAT) for use in primary care. J Public Health 2004, 26, 138-143, DOI:10.1093/pubmed/fdh132.

13. Downs, S.; Marquez, J. \& Chiarelli, P. The Berg Balance Scale has high intra- and inter-rater reliability but absolute reliability varies across the scale: a systematic review. J Physiother 2013, 59, 93-99, DOI:10.1016/S1836-9553(13)70161-9.

14. Cattelani, L.; Palumbo, P.; Palmerini, L.; Bandinelli, S.; Becker, C.; Chesani, F. \& Chiari, L. FRAT-up, a FallRisk Assessment Tool for Elderly People Living in the Community. J Med Internet Res 2015, 17, e41, DOI:10.2196/jmir.4064.

15. Bergmann, J.; Alexiou, C.; Howard, N. \& Smith, I., n.d. A normative data-set of Timed Up and Go component times under different conditions - Customer cases. Xsens 3D Motion Track. Available from: https://www.xsens.com/customer-cases/normative-data-set-timed-go-component-times-different conditions/ (accessed on 22 October 2015).

16. Greene, B.R.; O’Donovan, A.; Romero-Ortuno, R.; Cogan, L.; Scanaill, C.N. \& Kenny, R.A. Quantitative falls risk assessment using the timed up and go test. IEEE Trans Biomed Eng 2010, 57, 2918-2926, DOI:10.1109/TBME.2010.2083659.

17. Santos, A.; Guimarães, V.; Matos, N.; Cevada, J.; Ferreira, C. \& Sousa, I. 2015. Multi-sensor Exercise-based Interactive Games for Fall Prevention and Rehabilitation. IEEE, 2015, DOI:10.4108/icst.pervasivehealth.2015.259115.

18. Verghese, J.; Holtzer, R.; Lipton, R.B. \& Wang, C. Quantitative gait markers and incident fall risk in older adults. J. Gerontol. A Biol Sci Med Sci 2009, 64, 896-901, DOI:10.1093/gerona/glp033.

19. Hartikainen, S.; Lönnroos, E. \& Louhivuori, K. Medication as a Risk Factor for Falls: Critical Systematic Review. J. Gerontol. A Biol Sci Med Sci 2007, 62, 1172-1181. Available online: http://tinyurl.com/jclgokj (accessed on the 26 January 2016).

20. Guimarães, V.; Ribeiro, D. \& Rosado, L., 2013. A Smartphone-Based Fall Risk Assessment Tool: Measuring One Leg Standing, Sit to Stand and Falls Efficacy Scale, in: IEEE 15th International Conference on E-Health Networking, Applications and Services. Presented at the Healthcom, 2013, IEEE, Lisbon.

21. Tinetti, M.E.; Richman, D. \& Powell, L. Falls efficacy as a measure of fear of falling. J Gerontol 1990, 45, P239243, DOI: 10.1093/geronj/45.6.P239.

22. Tinetti, M.E. Performance-oriented assessment of mobility problems in elderly patients. J Am Geriatr Soc 1986, 34, 119-126, DOI: 10.1111/j.1532-5415.1986.tb05480.x. 
23. Beauchet, O.; Fantino, B.; Allali, G.; Muir, S.W.; Montero-Odasso, M. \& Annweiler, C. Timed up and go test and risk of falls in older adults: A systematic review. J Nutr Health Aging 2011, 15, 933-938, DOI:10.1007/s12603-011-0062-0.

24. Gunter, K.B.; White, K.N.; Hayes, W.C. \& Snow, C. Functional Mobility Discriminates Nonfallers From OneTime and Frequent Fallers. J Gerontol A Biol Sci Med Sci 2000, 55, M672-M676, DOI:10.1093/gerona/55.11.M672.

25. Rose, D. J.; Jones, J. C. \& Lucchese, N. Predicting the Probability of Falls in Community-Residing Older Adults Using the 8-Foot Up-and-Go: A New Measure of Functional Mobility. J Ageing Phys Act 2002, 10, 466-475. Available online: http://tinyurl.com/zb47m4y (accessed on the 18 February 2016).

26. Novaes, R.D.; Miranda, A.S. \& Dourado, V.Z. Usual gait speed assessment in middle-aged and elderly Brazilian subjects. Rev Bras Fisioter 2011, 15, 117-122. Available online: http://tinyurl.com/je2qpmn (accessed on the 19 February 2016).

27. Rehab Measures - Step Test. Rehabil. Meas. Database. n.d. Available online: http://www.rehabmeasures.org/Lists/RehabMeasures/DispForm.aspx?ID=1107 (accessed on 20 October 2015).

28. Whyatt, C.; Merriman, N. A.; Young, W. R.; Newell, F. N. \& Craig, C. A Wii bit of fun: a novel platform to deliver effective balance training to older adults. Games Health J 2015, 4(6), 423-433, DOI: 10.1089/g4h.2015.0006.

29. Clark, R. A.; Bryant, A. L.; Pua, Y.; McCrory, P.; Bennell, K. \& Hunt, M. Validity and reliability of the Nintendo Wii Balance Board for assessment of standing balance. Gait posture 2010, 31(3), 307-310, DOI:10.1016/j.gaitpost.2009.11.012.

30. Young, W.; Ferguson, S.; Brault, S. \& Craig, C. Assessing and training standing balance in older adults: a novel approach using the 'Nintendo Wii'Balance Board. Gait posture 2011, 33(2), 303-305, DOI:10.1016/j.gaitpost.2010.10.089.

31. Aguiar, B.; Silva, J.; Rocha, T.; Carneiro, S. \& Sousa, I. Monitoring physical activity and energy expenditure with smartphones, In: 2014 IEEE-EMBS International Conference on Biomedical and Health Informatics (BHI). Presented at the 2014 IEEE-EMBS International Conference on Biomedical and Health Informatics (BHI), valencis Spain, Jun 01- 04, 2014a, pp. 664-667, DOI:10.1109/BHI.2014.6864451.

32. Figo, D.; Diniz, P.C.; Ferreira, D.R. \& Cardoso, J.M. Preprocessing Techniques for Context Recognition from Accelerometer Data. Pers. Ubiquitous Comput 2010, 14, 645-662, DOI:10.1007/s00779-010-0293-9.

33. Aguiar, B.; Rocha, T.; Silva, J. \& Sousa, I. Accelerometer-based fall detection for smartphones, In: 2014 IEEE International Symposium on Medical Measurements and Applications (MeMeA). Presented at the 2014 IEEE International Symposium on Medical Measurements and Applications (MeMeA), ISCTE - University Institute of Lisbon, Portugal, Jun 11-12, 2014b. pp. 1-6, DOI:10.1109/MeMeA.2014.6860110.

34. Qiu, S.; Cai, X.; Ju, C.; Sun, Z.; Yin, H.; Zügel, M.; Otto, S.; Steinacker, J.M. \& Schumann, U. Step Counter Use and Sedentary Time in Adults: A Meta-Analysis. Medicine (Baltimore) 2015, 94, e1412, DOI:10.1097/MD.000000000000141.

(C) 2016 by the authors; licensee Preprints, Basel, Switzerland. This article is an open access article distributed under the terms and conditions of the Creative Commons by Attribution (CC-BY) license (http://creativecommons.org/licenses/by/4.0/). 